\title{
Curriculum vitae de los miembros de los Comités Editorial y Científico en orden alfabético
}

\section{Comité editorial}

Alejandro Castillejo. (Universidad de los Andes, Colombia). Doctor en Antropología Cultural, New School for Social Research, Departamento de Antropología (Estados Unidos). Magíster en Antropología Cultural, New School for Social Research, Departamento de Antropología (Estados Unidos). Magíster en Estudios sobre la Paz y el Conflicto, University for Peace and Conflict Studies (Austria). Especialista en Estudios en Conflicto y Desarrollo, Universidad JaumeI (España). Especialista en Estudios sobre el Conflicto y la Paz, University for Peace and Conflict Studies (Austria). Antropólogo, Universidad Nacional de Colombia. Ha trabajado como consultor de la Comisión Peruana de la Verdad, profesor visitante en la Universidad de Londres, School of Oriental and African Studies (SOAs) (Reino Unido), Zayed University (Emiratos Árabes), del Instituto de Estudios Avanzados en Berlín (Alemania) e investigador de las Universidades de Columbia y Pensilvania (Estados Unidos) y del Instituto para la Justicia y la Reconciliación. En la actualidad trabaja como profesor asociado de la Facultad de Ciencias Sociales, Departamento de Antropología, Universidad de los Andes (Colombia). 
Roddy Brett. (Universidad del Rosario, Bogotá, Colombia). Ph. D. in Politics, Queen Mary College, University of London. Magíster en Antropología Social, University of Cambridge, Jesus College. Profesor titular de la Facultad de Ciencia Política, Gobierno y Relaciones Internacionales, Universidad del Rosario, Bogotá, Colombia. En sus investigaciones ha trabajado temas relacionados con derechos humanos, historia de conflictos armados en América Latina y movimientos sociales. Entre sus publicaciones más recientes se encuentra: La voluntad indómita: teorías de los movimientos sociales y la acción colectiva (2012), coeditado con Freddy Cante. El movimiento maya diez años después de los acuerdos de paz: retos y posibilidades, (2010), coeditado con Santiago Bastos.

Raimund Krämer. (Universidad de Potsdam, Alemania). Doctor en Estudios de Ciencias Políticas y Relaciones Internacionales en el Instituto de Relaciones Internacionales en Potsdam. Profesor universitario de Política Internacional y Estudios Comparativos en la Universidad de Potsdam. Director de la revista WeltTrends (Cuaderno de Politica Internacionaly Estudios Comparativos). Los temas de su trabajo de investigación han sido: regionalismo y el orden del mundo, historia de la diplomacia, regiones como actores internacionales, federalismo y relaciones internacionales, fronteras y cooperación transfronteriza, élites y política exterior, autoritarismo como fenómeno global, militares en América Latina y gobernar en la Unión Europea. Entre sus publicaciones se encuentra: Der Aufstand des Jahres 1432. Aktuelle Umbrüche im Nahen Osten und die Weltpolitike (en coautoría con Lutz Kleinwächter) (2011). WeltTrends Spezial, 1; Wissenschaftliches Schreiben (2011) y "Autoritarismus global. Überlegungen zu einem aktuellen politischen Phänomen" (2012) WeltTrends 82.

Edgard Moncayo Jiménez. (Universidad Central de Colombia, Colombia). Doctorando en Ciencias Económicas, Universidad Nacional de Colombia. Magíster de la Escuela de Gobierno de la Universidad de Harvard y del Departamento de Estudios Latinoamericanos de la Universidad de Georgetown (Estados Unidos). Economista. Es miembro de número de la Academia Colombiana de Ciencias Económicas. Dirigió los trabajos de la Misión Bogotá Siglo xxi, que fueron pioneros en la prospectiva urbana de la capital. Trabajó como asesor regional del 
Ilpes-Cepal. En la actualidad es director del Centro de Investigación y Estudios de la Facultad de Ciencias Administrativas, Económicas y Contables de la Universidad Central de Colombia.

Dieter Nohlen. (Heidelberg University, Alemania). Politólogo e historiador, universidades de Colonia y Heidelberg (Alemania). Profesor titular emérito de la Universidad de Heidelberg. Autor de un sinnúmero de libros en alemán, inglés y castellano, con traducciones a otros idiomas.

Julieta Rostica. (Universidad de Buenos Aires, Argentina). Doctora en Ciencias Sociales y Humanas, Universidad de Quilmes (Argentina). Magíster en Estudios Latinoamericanos: diversidad cultural y complejidad social, Universidad Autónoma de Madrid (España). Licenciada en Sociología con título de honor por la Universidad de Buenos Aires. Fue auxiliar de investigación en los siguientes proyectos colectivos: Nación, ciudadania y derechos bumanos en los países del Mercosur (2001-2004), Los sonidos del silencio. Dictaduras y resistencias en América Latina, 1964-1989 (2004-2007) y Las condiciones sociohistóricas de la democracia y la dictadura en América Latina 1954-2010 (2008-2010), del Instituto de Investigaciones Gino Germani, dirigidos por el doctor Waldo Ansaldi y por la doctora Patricia Funes y subsidiados por la Secretaría de Ciencia y Técnica de la Universidad de Buenos Aires. Fue becaria categoría Estímulo de la Secretaría de Ciencia y Técnica de la Universidad de Buenos Aires; becaria doctoral del Consejo Nacional de Investigaciones Científicas y Técnicas (Conicet); becaria de Maestría de la Fundación Carolina (España) y en la actualidad becaria posdoctoral de Conicet. Es docente de las cátedras Historia Social Latinoamericana y del taller de Sociología Histórica de América Latina del programa de Sociología de la Facultad de Ciencias Sociales de la Universidad de Buenos Aires.

Rubén Sánchez. (Universidad del Rosario, Colombia). Doctor en Ciencias Económicas, École Pratique des Hautes Études, Universidad de París (Francia). Se desempeña como profesor en las Facultades de Ciencia Política y Gobierno y de Relaciones Internacionales de la Universidad del Rosario e investigador del Centro de Estudios Políticos e Internacionales (CEPI) de la misma Universidad. 
Victoria Sanford. (Lebman College-City University of New York, Estados Unidos). Ph. D. in Anthropology, Stanford University (Estados Unidos). M. A. in Anthropology, Stanford University (Estados Unidos). M. A. in Society and Culture of Central America, San Francisco State University (Estados Unidos). B. A. in Social Science with concentration in Economics, California State University Sacramento (Estados Unidos). Ha encaminado su trabajo académico y sus investigaciones hacia los temas relacionados con los derechos humanos. Ha trabajado como asesora de múltiples ONG y fundaciones que trabajan en temas relativos a derechos humanos y justicia transicional. En el campo académico se ha desempeñado como investigadora y profesora asociada en varias universidades de Estados Unidos. En la actualidad es profesora titular de Antropología en el Departamento de Antropología, Lehman College-City University of New York. Miembro doctoral de Facultad, Departamento de Antropología, The Graduate Center, The City University of New York (Estados Unidos). Investigadora asociada del Center for International Conflict Resolution, Columbia University (Estados Unidos). Afiliada académica, Center for the Study of Genocide and Human Rights, Rutgers University (Estados Unidos).

Zidane Zeraoui. (Instituto Tecnológico y de Estudios Superiores de Monterrey [ITESM], México). Doctor en Ciencia Política, unam (México). Magíster en Relaciones Internacionales, La Sorbona (Francia) y en Ciencia Política, unam. Licenciado en Comunicación, Universidad de Argel (Argelia). Miembro del Sistema Nacional de Investigadores (SNIConacyt) de México, nivel 2; profesor investigador del ITESM, campus Monterrey, Departamento de Relaciones Internacionales; coordinador de Internacionalización de la División de Humanidades y Ciencias Sociales del ITESM. 


\section{Comité Científico}

Mauricio Archila Neira. (Universidad Nacional de Colombia, Colombia). Ph. D. en Historia, State Universtity of New York (Stony Brook) (Estados Unidos). Magíster en Economía y Recursos Humanos, Pontificia Universidad Javeriana (Colombia). Licenciado en Filosofía y Letras, con especialización en Historia, Pontificia Universidad Javeriana (Colombia). Ha sido profesor de la Universidad Nacional de Colombia desde 1978 en el Departamento de Historia, profesor visitante en el Instituto de Estudios Iberoamericanos (Ilais) de Columbia University, Nueva York (Estados Unidos). Ha trabajado como investigador en diversas ocasiones con el Centro de Investigación y Educación Popular (Cinep).

Ana María Bejarano. (University of Toronto, Canadá). Doctora en Ciencias Políticas de la Universidad de Columbia (Estados Unidos). Politóloga. Profesora asistente del Departamento de Ciencia Política de la Universidad de Toronto (Canadá). Autora de varios artículos sobre Ciencia Política en América Latina y Canadá.

Marta Elena Casaús Arzú. (Universidad Autónoma de Madrid, España). Doctora en Ciencias Políticas y Sociología, Universidad Complutense de Madrid (España). Licenciada en Ciencias Políticas y Sociología, Universidad Complutense de Madrid (España). Ha trabajado como profesora e investigadora en diferentes universidades de Guatemala y España. Se desempeña como profesora titular de Historia de América y directora de la Maestría Europea en Estudios Latinoamericanos en la Universidad Autónoma de Madrid. Ha sido investigadora principal en múltiples proyectos relacionados con procesos de cambio social e integración en América Central, participación de la sociedad civil en la construcción del Estado democrático en América Central, construcción de la Nación e imagen del indio en el pensamiento político del siglo xIx, elecciones y partidos políticos en América Latina, la construcción de la Nación y la visión del indio en el pensamiento político de los pensadores centroamericanos del siglo XIX, las élites intelectuales centroamericanas: su imaginario de Nación y la visión del indio (1920-1990), las redes intelectuales iberoamericanas y la pro- 
yección del hispanismo y el regeneracionismo en el espacio americano y algunos conceptos vertebradores de la modernidad en América Latina, entre otros.

Michael Gold-Biss. (National Defense University, Estados Unidos). Doctor en Relaciones Internacionales de la American University (Estados Unidos). EBA de la Universidad de Minnesota (Estados Unidos). Magíster en Ciencia Política de la Universidad de Delaware (Estados Unidos). B. A. en Asuntos Internacionales e Historia de LaFayette College (Estados Unidos). Internacionalista estadounidense. Ha sido asistente del vicepresidente para programas internacionales en el A\&M International University en Laredo, Texas (Estados Unidos). Ha sido director del Programa de Relaciones Internacionales en la Universidad Estatal de Saint Cloude (Estados Unidos). Hoy en día es profesor en Asuntos de Seguridad Nacional del Centro Hemisférico de Defensa (CDs) de la Universidad Nacional de Defensa en Washington, D. C. (Estados Unidos).

Charles R. Hale. (University of Texas at Austin, Estados Unidos). Ph. D., Stanford University (Estados Unidos). Trabaja como profesor e investigador en el Departamento de Antropología y director del Teresa Lozano Long Institute of Latin American Studies (LLILAS), University of Texas at Austin (Estados Unidos). Sus áreas de interés se enmarcan dentro de la raza/etnicidad, identidades políticas, concientización y resistencia, antropología activista, antropología social, América Latina y el Caribe.

Bernard Labatut. (Institut d'Etudes Politiques de Toulouse, Francia). Doctor en Ciencia Política (Cum Laude), Universidad de Ciencias Sociales de Toulouse (Francia). Su disertación se titula La política de defensa de la España democrática. Magíster en Derecho Público, mención Derecho Internacional, Facultad de Derecho, Universidad de Ciencias Sociales de Toulouse. Se desempeña como profesor de Ciencia Política y Relaciones Internacionales en la Universidad de Ciencias Sociales e Instituto de Estudios Políticos de Toulouse (Francia). 
Louis Valentín Mballa. (Instituto Tecnológico y de Estudios Superiores de Monterrey [ITESM], campus Querétaro, México). Doctor en Ciencias Políticas y Sociales con Orientación en Relaciones Internacionales de la UNAM (México). Magíster en Estudios en Relaciones Internacionales de la unAm (México). Licenciado en Filosofía. Recientemente ha comenzado sus estudios de posdoctorado en Relaciones Internacionales en la Universidad de Pretoria (Sudáfrica). Trabajó hasta hace poco como profesor de planta en el ITESM (campus Querétaro), de materias relacionadas con las teorías de las relaciones internacionales, fundamentos de la ciencia política y relaciones de África con el mundo. También ha realizado actividades de apoyo académico en el Departamento de Becas del Programa de Posgrado en Ciencias Políticas y Sociales de la UNAM.

Jenny Pearce. (University of Bradford, Reino Unido). Profesora de Estudios Políticos Latinoamericanos en el Departamento de Estudios de Paz de la Universidad de Bradford (Reino Unido). Se desempeña como directora del Centro para los Estudios de Participación en la misma universidad. Ha concentrado su investigación en los temas de cambio social y democratización en América Latina. Otros temas de su interés son: sociedad civil, mecanismos de acción colectiva y participación, reconstrucción posconflicto y desarrollo, violencias complejas y pobreza. Es autora de los libros Promised Land: Peasant Rebellion in Chalatenango, El Salvador (1985) y Colombia: Inside the Labyrinth (1990).

Luz del Socorro Ramírez. (Colombia). Doctora en Ciencia Política, Departamento de Ciencia Política, Universidad Sorbona, París (Francia). Doctora en Ciencia Política de la Universidad Central de Venezuela. Magíster en Análisis de Problemas Políticos, Económicos e Internacionales Contemporáneos, Instituto de Altos Estudios para el Desarrollo, Universidad Externado de Colombia. Ha sido coordinadora en Colombia del Grupo Académico Colombia-Venezuela desde 1998 y promotora del proyecto académico andino-brasileño desde 2003. Trabajó como profesora titular del Instituto de Estudios Políticos y Relaciones Internacionales (Iepri), Universidad Nacional de Colombia desde 1992. 
Javier Roiz Parra. (Universidad Complutense de Madrid, campus de Somosaguas, España). Doctor en Ciencias Políticas y Sociología. Trabajó como docente e investigador visitante en Princeton University (Estados Unidos), Sigmund Freud Institut de Fráncfort (Alemania), Wesleyan University (Estados Unidos), Saint Louis University (Estados Unidos), Universidad Central de Venezuela-Cipost y Universitat Rovira i Virgili (España). Es director de la revista académica Foro Interno y profesor e investigador del Departamento de Ciencia Política y de la Administración II, Facultad de Ciencias Políticas y Sociología, Universidad Complutense de Madrid (España).

Jinseog Yu. (Sookmyung Women's University, Seoul, Korea). Ph. D. en Ciencia Política, Duke University (Estados Unidos). Magíster en Ciencia Política, Yonsei University (Corea). Profesor asociado del Departamento de Ciencia Política y Relaciones Internacionales, Sookmyung Women's University. Ha realizado distintas publicaciones, entre ellas: "Domestic Politics and Foreign Policy", en Kye-dong Kim et al. (Eds.), (2012). Contemporary Foreign Policy 2nd edition (Seoul: Myung-in). "The Korean War and U. S. Nuclear Strategy in the Formative Years of Nuclear Deterrence". (2011). Korea \& World Politics, summer. "Study of International Relations in Korea: Reality and Search for an Alternative" (en coautoría con Kun Young Park). (2008). Journal of Asia-Pacific Studies, 15 (1). "Carrots and Sticks in Non-proliferation Policy: Case Studies and their Implications for North Korea's Nuclear Program". (2008). 21st Century Political Science Review, 18, (1). "Convergence and Divergence of U. S. and South Korean Strategies toward North Korea’s Nuclear Program” (2005). Korea Observer, 39 (2). 